九州大学学術情報リポジトリ

Kyushu University Institutional Repository

\title{
A Detection Method for Multi-Touch on Single- Layered Capacitive Touch Panel
}

Kyoung, Kyuwon

Interdisciplinary Graduate School of Engineering Sciences, Kyushu University

Yoneda, Ryota

Interdisciplinary Graduate School of Engineering Sciences, Kyushu University

Hattori, Reiji

Art, Science and Technology Center for Cooperative Research, Kyushu University

https://doi.org/10.5109/1440972

出版情報: Evergreen. 1 (1)，pp.20-24，2014-03. Green Asia Education Center バージョン：

権利関係 : 


\title{
A Detection Method for Multi-Touch on Single-Layered Capacitive Touch Panel
}

\author{
Kyuwon Kyoung $^{*}$, Ryota Yoneda ${ }^{1}$, Reiji Hattori ${ }^{2}$ \\ 1 Interdisciplinary Graduate School of Engineering Sciences, Kyushu University, Japan \\ 2 Art, Science and Technology Center for Cooperative Research, Kyushu University, Japan \\ *Author to whom correspondence should be addressed, \\ E-mail: kyoung@astec.kyushu-u.ac.jp
}

(Received February 5, 2014; accepted March 7, 2014)

\begin{abstract}
We have devised a method that employs surface- and self-capacitive sensing principles for touch detection with a single-layered capacitive touch panel. In this method, a single electrode layer consisting of a stripe-shaped one-dimensional electrode array was used to enable multi-touch detection, and a capacitive sensing circuit that uses a trans-impedance amplifier was selected to simplify the circuit structure of the touch panel. The results indicate that the number of the panel fabrication processes and the amount of the touch electrode material required can be reduced by half by using the proposed method.
\end{abstract}

\section{Introduction}

Due to their high demand in the electronic industry, competition for usage of rare earth metals has recently intensified $^{1)}$. As such, attempts to replace these metals with other materials such as organic conductor polymers or carbon nanotubes have been actively researched. A good example of this paradigm is the one used in touch panel technology ${ }^{2}$.

Capacitive touch panels are in current widespread usage as input devices in mobile applications such as smartphones, tablets, and notebook PCs because of their good optical performance, strong durability, and easy system integration $^{3)}$.

In these panels, the touch positions are determined by detecting changes in floating capacitance induced by a finger touch on the touch panel. Depending on the sensing methods, the capacitive touch panel classifications include surface, self-capacitive, and mutual capacitive types ${ }^{4}$. Figure 1 shows the configuration of touch electrodes and an equivalent circuit for each method.

The surface type calculates the touch positions from a ratio of currents at each side of the touch panel. This type is suitable for use in low-cost touch panel because it is composed of only a single-layered electrode sheet. However, the ratio used in touch detection limits the multi-touch function required for mobile devices ${ }^{5,6)}$.

By contrast, the self-capacitive and mutual capacitive types calculate the touch positions based on changes of the capacitive coupling at each electrode. These types are advantageous for realizing the multi-touch function. The touch-electrode array in the vertical and horizontal directions, however, increases the composition process of the touch panel and raises its $\operatorname{cost}^{7,8)}$.

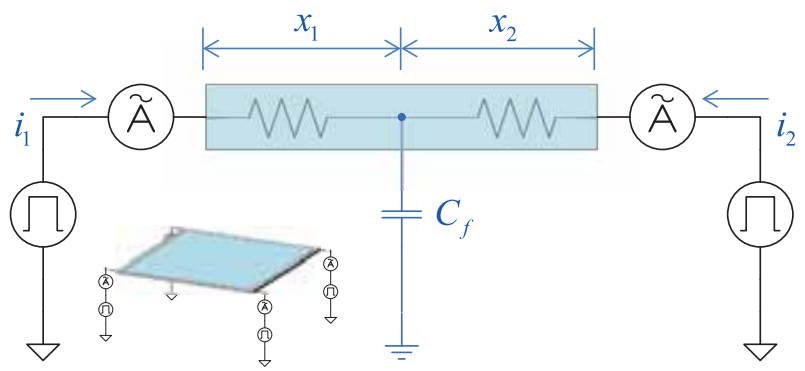

(a) Surface type

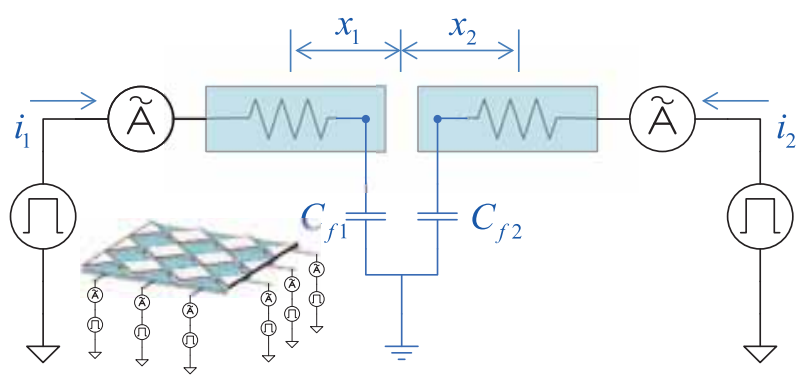

(b) Self-capacitive type

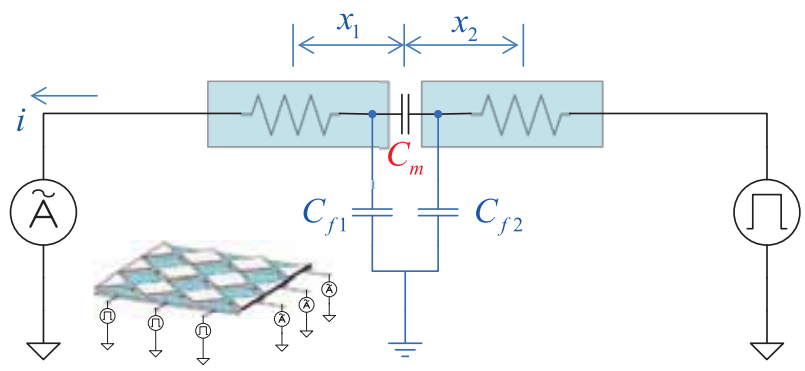

(c) Mutual capacitive type

Fig. 1 Configuration of touch electrodes and an equivalent circuit in each method. 
Considering this information, we undertook studies ${ }^{9,10)}$ to investigate whether the surface and self-capacitive types can be unified to enable multi-touch detection on a single-layered touch panel.

In this study, a touch panel that consists of stripe-shaped one-dimensional electrode array was prepared with a single electrode layer to detect the multi-finger touch. Also, two types of current-sensing circuits were evaluated by Simulation Program with Integrated Circuit Emphasis (SPICE) simulation for adopting a suitable one to the proposed touch panel. From a demonstration kit, we verified that the proposed method can reduce the use of electrode material and the fabrication process of the touch panel while maintaining the function of multi-touch detection.

\section{Methodology}

\subsection{Capacitive touch panel with stripe-shaped electrodes}

Figure 2 (a) shows the panel layout of a 4-in touch panel, in which a vertical electrode layer of the touch

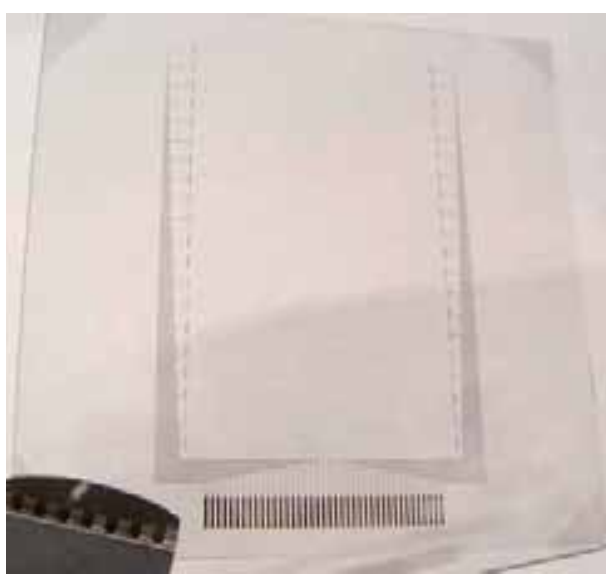

(a) Panel layout
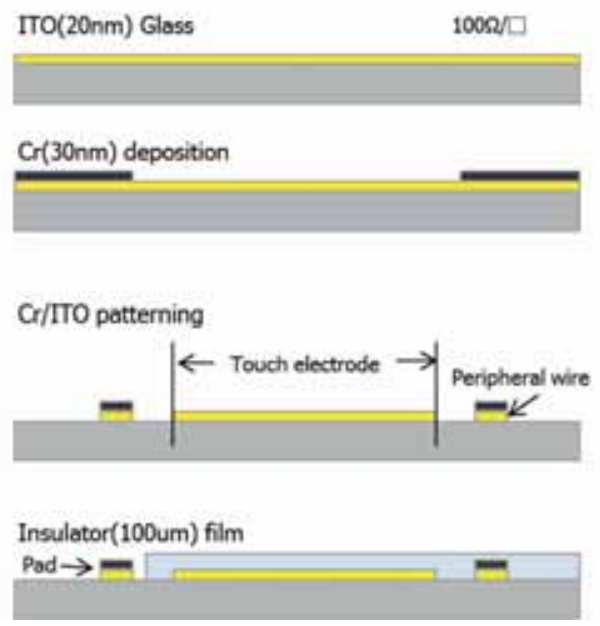

(b) Composition process

Fig. 2 Capacitive touch panel with stripe-shaped electrodes. panel could be omitted by adopting stripe-shaped electrodes.

The 4-in touch panel consisted of 20 stripe-shaped electrodes with connections used as channels on both sides. The width and length of each electrode were 3.6 $\mathrm{mm}$ and $54 \mathrm{~mm}$, respectively, and the pitch between the electrodes was $3.8 \mathrm{~mm}$.

Figure 3 (b) shows the composition process of a touch panel fabricated from a $20 \mathrm{~nm}$ indium tin oxide (ITO) evaporated glass substrate. First, a $30 \mathrm{~nm}$ chromium (Cr) layer was deposited through an evaporation process to decrease the resistance of the peripheral wire. At that time, the region of the touch panel was masked with photoresist to prevent coverage by metal, thus assuring that the area remained transparent. Next, the $\mathrm{Cr}$ and ITO layer was patterned by photolithography and wet etching. Finally, a $100 \mu \mathrm{m}$ insulator film was laminated on the top of the substrate.

\subsection{Principle of the touch panel}

Figure 3 shows the principle of the proposed touch panel. A one-dimensional array of ITO electrodes is connected to the driving circuit at both edges and applies a sine-wave voltage with an adequate frequency. When a finger approaches the electrodes, some currents flow into the floating capacitance formed between the finger and electrodes.

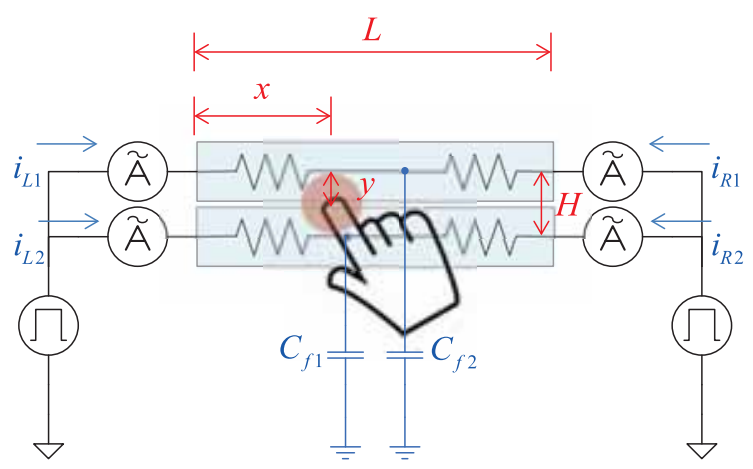

Fig. 3 Equivalent circuitry of the proposed method.

Because the current values are inversely proportional to the resistances between the edges and the touching point, the touching position in the $\mathrm{x}$-direction is given by Eq. (1), according to the principle of one-dimensional surface capacitive sensing.

$$
x=\frac{R_{L 1}}{R_{L 1}+R_{R 1}} \cdot L=\frac{i_{R 1}}{i_{L 1}+i_{R 1}} \cdot L
$$

Conversely, the vertical position between the adjacent electrodes can be determined by the ratio of the floating capacitance induced on each electrode according to Eq. (2). This phenomenon is based on the principle of self-capacitive sensing. 


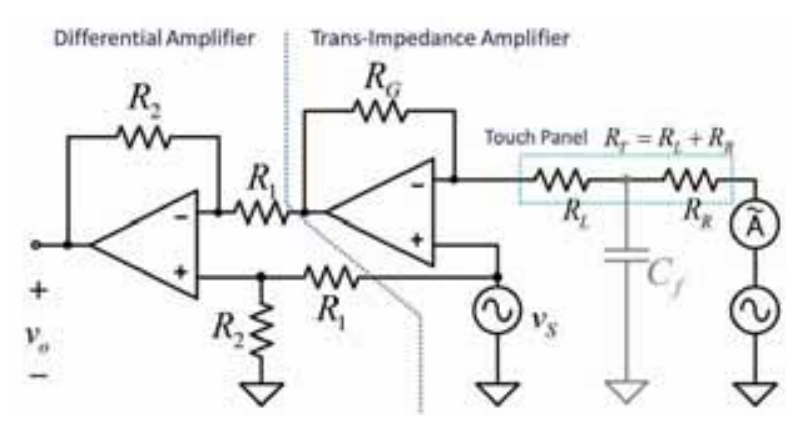

(a) With a TIA

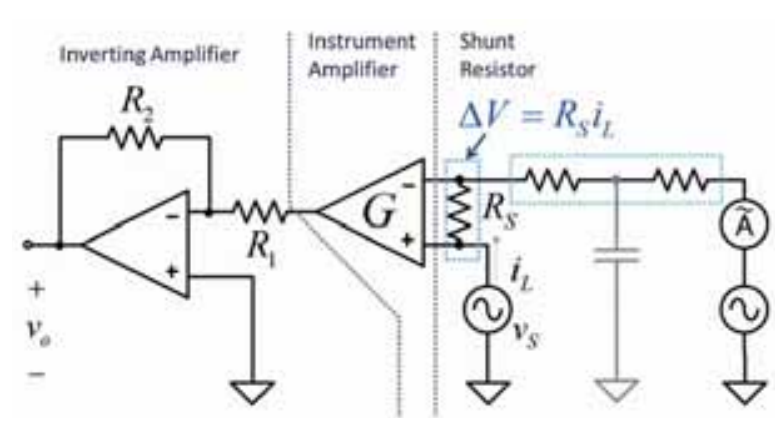

(b) With a shunt resistor and an instrument amplifier

Fig. 4 Current sensing circuits for the proposed touch panel.

$$
y=\frac{C_{f 1}}{C_{f 1}+C_{f 2}} \cdot H=\frac{i_{R 1}}{i_{R 1}+i_{R 2}} \cdot H
$$

Under this principle, multi-touch sensing is available, except for the touch positions exist on the same electrode.

\section{Results and discussion}

\subsection{Current sensing circuit}

To measure the current between the source of the touch signal and the floating capacitance, it is necessary to use a high-side current sensing method.

As shown in Figure 4, two types of the high-side current sensing circuit can be considered at the front-end terminal in the capacitive touch panel ${ }^{11)}$. Figure 4 (a) shows a current sensing circuit with a trans-impedance amplifier (TIA). This circuit detects changes in the current based on the difference between the signal passing through the TIA and the touch signal from an oscillator. Figure 4 (b) shows a current sensing circuit with a shunt resistor and an instrument amplifier. This

Table 1 Output characteristics of current sensing circuits.

\begin{tabular}{cc}
$\begin{array}{c}\text { Current sensing circuit } \\
\text { with a TIA }\end{array}$ & $\begin{array}{c}\text { Current sensing circuit } \\
\text { with an Inst. Amp }\end{array}$ \\
\hline$\left|v_{o}\right|=\frac{R_{2}}{R_{1}} 2 \sin \frac{\phi}{2}\left|v_{s}\right|$ & $\left|v_{o}\right|=\frac{R_{2}}{R_{1}} G|\Delta V|$
\end{tabular}

Where,

Where,

$$
\begin{array}{cl}
\phi \approx \tan ^{-1} \frac{\omega R_{G} R_{R} C_{f}}{R_{T}} \quad \approx \frac{\omega\left(R_{S}+R_{R}\right) C_{f}}{\left(2 R_{S}+R_{T}\right)}\left|v_{s}\right|
\end{array}
$$

circuit detects changes in voltage drop at the shunt resistor.

As shown in Table 1, the output characteristics of the

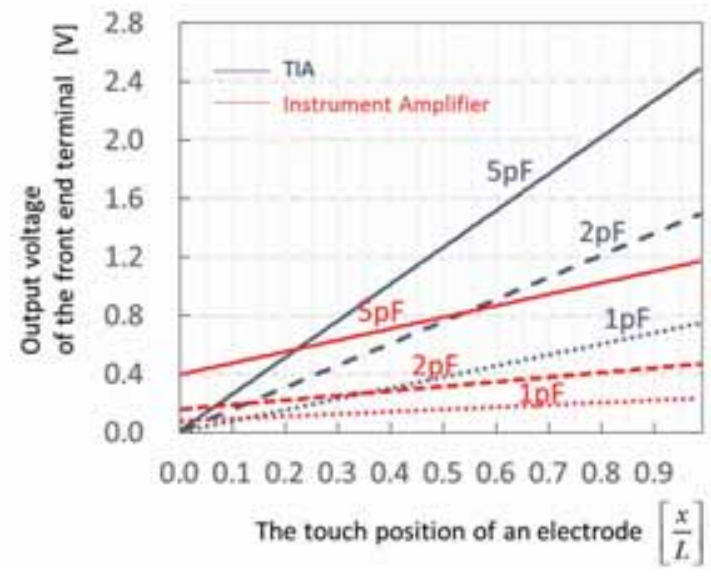

(a) Variation in floating capacitance at the touch area

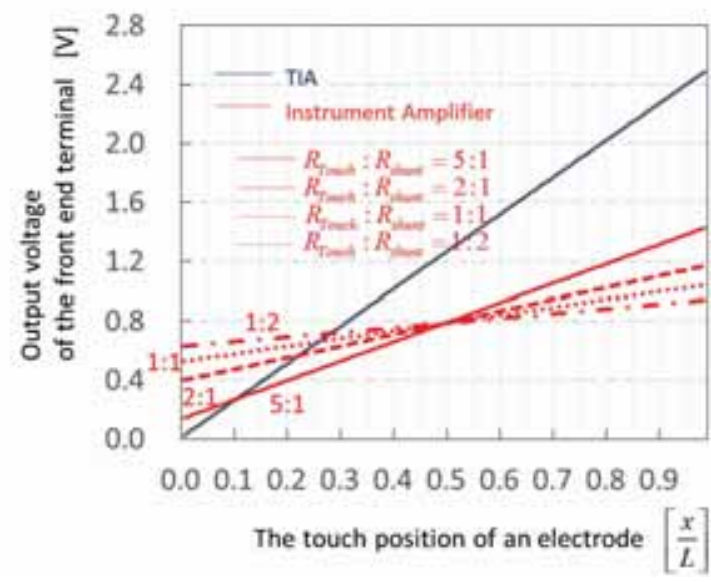

(b) Variation in the ratio of the resistances between the shunt resistor and the touch electrode

Fig. 5 Output voltage of current sensing circuits. 


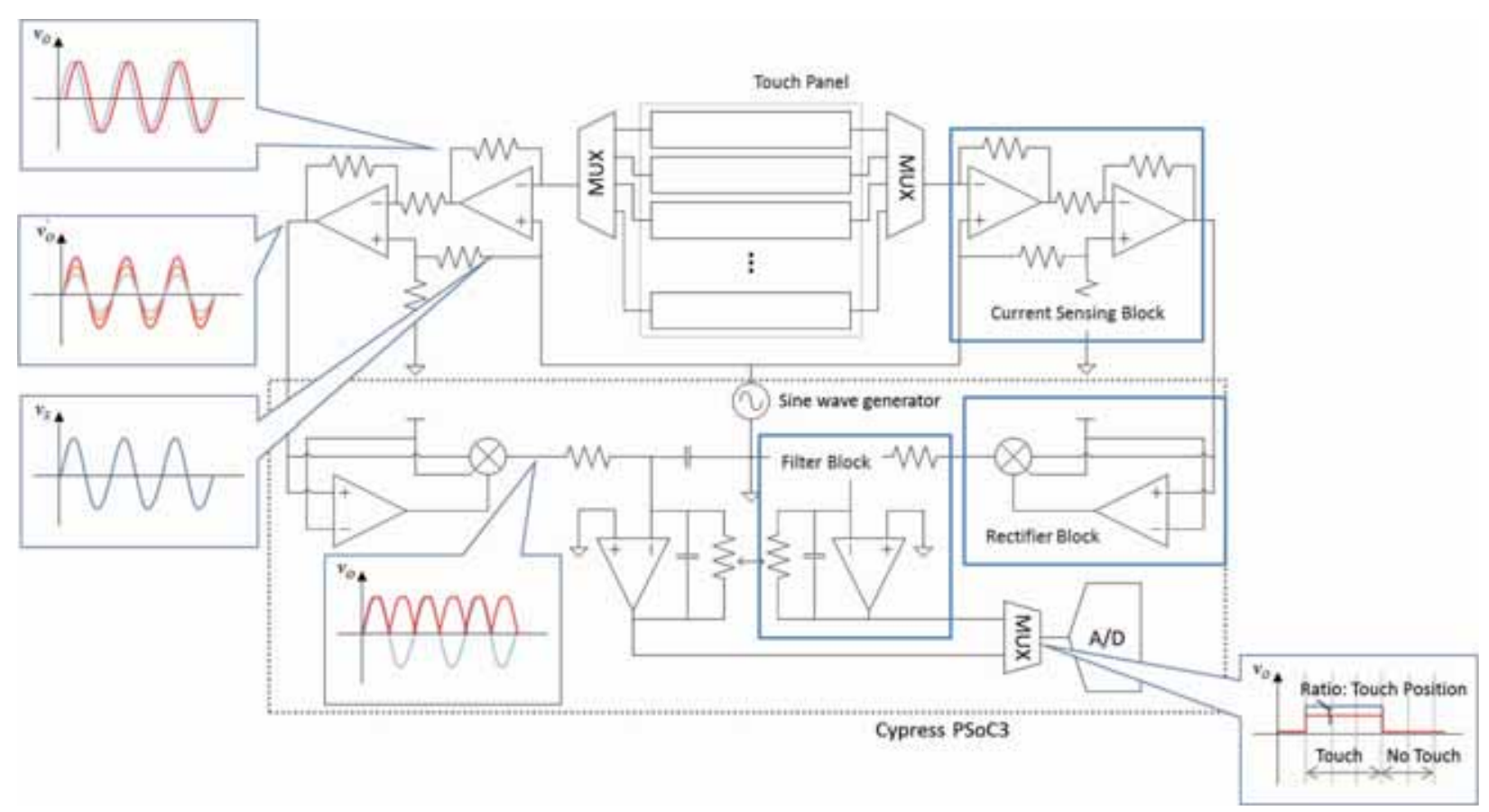

Fig. 6 Block diagram of the proposed circuit board.

front-end terminal in each circuit were derived such that these circuits are connected on both ends of the electrode of the proposed panel, where $R_{T}$ is the resistance of a touch electrode, $R_{R}$ is the resistance of the touch electrode on the right side of a touch position, $C_{f}$ is the floating capacitance by touch, and $\omega$ is the angular velocity of the touch signal.

Figure 5 shows the SPICE results of the current sensing circuits. Figure 5 (a) shows the output voltage for the floating capacitances, and Figure 5 (b) shows the output voltage for the ratio of the resistances between the shunt resistor and the touch electrode.

As a result, three problems are apparent in the current sensing circuit with an instrument amplifier. First, because of the lack of a feedback path, the input current of the amplifier should be small by using an instrument amplifier, which is more expensive than a common amplifier. Second, the resistance of the touch electrode should be higher than that of the shunt resister, in order to expand the dynamic range of touch detection, whereas the potential voltage is proportional to the resistance of the shunt resistor. Third, the amplifier has a low cut-off frequency and requires an additional amplifier to increase the gain.

These results indicate that the circuit with the TIA is more suitable for use as a current sensing circuit of the proposed touch panel than that with the instrument amplifier.

\subsection{Design of circuit board}

Figure 6 shows the block diagram of the proposed method. The circuit board consists of a sine wave generator, current sensing blocks, rectifier blocks, low pass filter blocks, and an analog-to-digital (A/D) converter. The current sensing blocks were directly embedded into the board by using operational amplifier integrated circuits (ICs), and the rectifier block was embedded into the board with a Cypress PSoC3 IC.

The touch signals from the sine wave generator were applied to each end of the touch electrode. Then, the output voltages of the differential amplifiers were varied near the touch position. Next, the alternating current (AC)

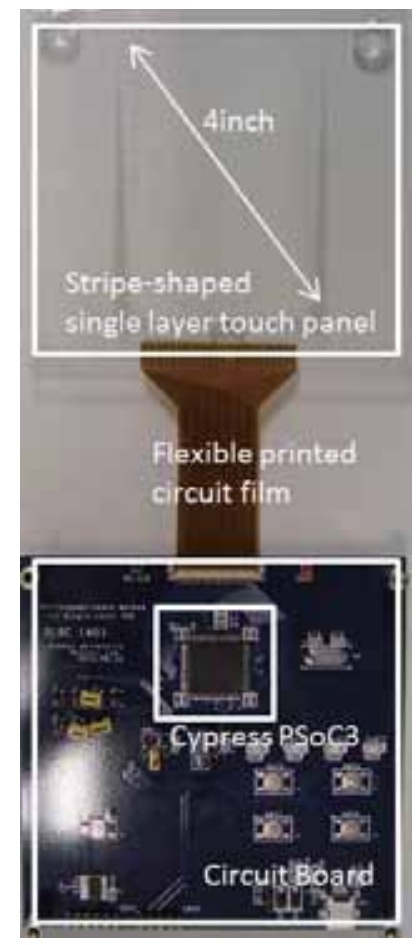

Fig. 7 Demonstration kit of the proposed method. 
outputs were rectified by the rectifier block. Finally, the direct current (DC) outputs, whose ratio determined the touch positions for $\mathrm{A} / \mathrm{D}$ conversion, were prepared through the low pass filter blocks.

\subsection{Demonstration}

Figure 7 shows a demonstration kit that consists of a 4-in stripe-shaped single-layered touch panel and a circuit board. A flexible printed circuit film was attached by an anisotropic conductive film (ACF). The sheet resistances of the touch electrode and the peripheral wire were $98.4 \Omega / \square$ and $2.4 \Omega / \square$, respectively.

Figure 8 shows an example of the distribution of output voltages from two finger touches.

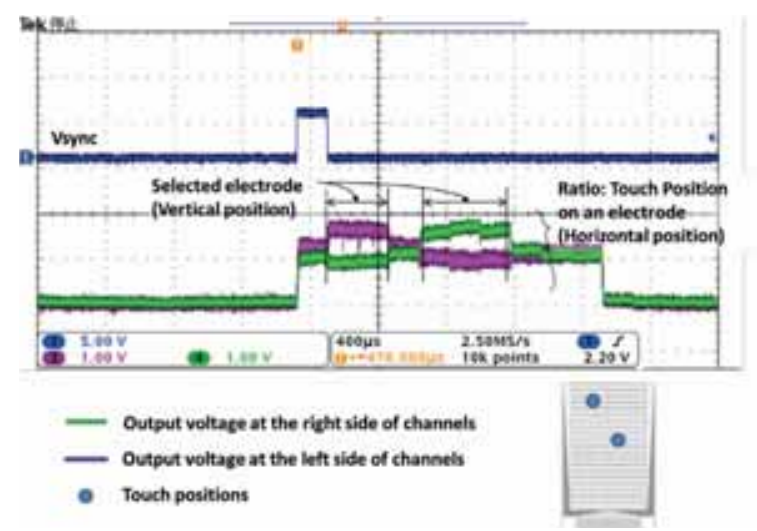

Fig. 8 A distribution of output voltages by two finger touches.

The horizontal touch position on an electrode was determined from the ratio of the output voltages between the right and left sides of the electrode. In addition, the vertical touch position on the adjacent electrodes was determined from the ratio of the output voltages between the channels. The results indicate that the proposed method for realizing multi-touch function on a single layered electrode was verified.

The specifications of the demonstration kit are summarized in Table 2.

Table 2 Specification of the demonstration kit.

\begin{tabular}{cc}
\hline Item & Specification \\
\hline \hline Touch Panel Size & 4inch diagonal \\
Number of Electrode layer & 1 layer \\
Number of Channel & 20 channels \\
Scan Rate & $100 \mathrm{~Hz}$ \\
S/N & $10 \sim 15 \mathrm{~dB}$ \\
Number of touches & Multi-touch \\
\hline
\end{tabular}

\section{Conclusion}

We proposed a detection method for multi-touch on a single-layered capacitive touch panel. In this method, the principles of the surface capacitive touch sensing and the self-capacitive sensing types were unified to detect multi-touch on a single-layered electrode. The proposed method was verified by using a demonstration kit.

The amount of electrode material and the number of composition processes of the touch panel could be halved by omitting the vertical electrode layer while maintaining the function of multi-touch detection.

\section{References}

1) Y. Kanazawa and M. Kamitani, Proceedings of Rare Earths '04 (Journal of Alloys and Compounds, 408-412), 1339-1343, Nara (2006).

2) S. Bae, H. Y. Lee, X. Xu, J. Park, Y. Zheng, J. Balakrishnan, T. Lei, H. Kim, Y. Song, Y. Kim, K. Kim, B. Özyilmaz, J. Ahn, B. Hong and S. Iijima, Nature Nanotechnology, 5, 574-578 (2010).

3) G. Barrett and R. Omote, Information Display, 26(3), 16-21 (2010).

4) J. Colegrove, Information Display, 26(3), 22-24 (2010).

5) H. Haga, J. Yanase, Y. Kamon, K. Takatori, H. Asada and S. Kaneko, Society for Information Display 2010 Digest of Technical Papers, 45-1, 669-672 (2010).

6) K. Kyoung, K. Yuge and R. Hattori, Proceedings of the 11th International Meeting on Information Display, 23-2, 174-175, Seoul (2011).

7) T. Wang and T. Blankenship, Information Display, 27(3), 8-11 (2011).

8) D. Lee, Information Display, 27(3), 12-16 (2011).

9) K. Kyoung and R. Hattori, Proceedings of the 19th International Display Workshops, INPp-2, 315-318, Kyoto (2012).

10) R. Hattori, K. Kyoung and R. Yoneda, Proceedings of the 20th International Display Workshops, INP3-1, 1626-1629, Sapporo (2013).

11) R. Yoneda, K. Kyoung and R. Hattori, Proceedings of the 2013 IEICE Society Conference, C-9-10, Fukuoka (2013). 